\title{
Episodic Memory for Autonomous Agents
}

\author{
T. Deutsch $\dagger$, Member, IEEE, A. Gruber $\dagger, \mathrm{R}$. Lang $\dagger$, Member, IEEE, and R. Velik $\dagger$, Member, IEEE \\ $\dagger$ Institute of Computer Technology of the Vienna University of Technology, Vienna, Austria \\ \{deutsch, langr, velik\}@ict.tuwien.ac.at, and.gruber@gmx.net
}

\begin{abstract}
Knowledge about already experienced situations improves the quality of decision making. The outcomes of decisions in the past can be evaluated and used as a feedback mechanism. This enables the decision making unit is able to learn from past situations. In neuro-psychanalysis, this kind of data storage is called episodic memory. This work describes an episodic memory architecture derived from theories from psychology and neuropsychoanalysis. The intended applications for this architecture are autonomous agents and building automation systems. The results of the simulation with the autonomous agents have shown that agents with the episodic memory adapt faster to new situations than memory-less agents.
\end{abstract}

Keywords-Episodic Memory, Decision Making, Autonomous Agents

\section{INTRODUCTION}

In the near future, building automation systems will be equipped with hundreds of thousands of sensors, and it is very likely that current control systems will not be able to deal with this complexity [1]. The Artificial Recognition System (ARS) project $^{1}$ [2], [3] aims to find new approaches to control building automation systems. The developed model is divided into two parts: ARS-PC (PerCeption) and ARS-PA (PsychoAnalysis).

The first one deals with transforming sensor data into symbols. Thus, its goal is to reduce the amount of data while increasing the meaning of them [4]. Three layers of symbols are defined [5]. The micro-symbol layer represents more or less the sensor readings. The next layer is the snapshot symbol layer. Here, the micro-symbols are combined to fewer, but more meaningful symbols-the snapshot-symbols (for example tactile floor sensors and motion sensors are combined into the symbol person). The top-layer-representation layercontains long lasting symbols-the representation symbols. Person A entering a room, walking around, and leaving it again would be such a symbol.

The second part of the model is a novel interdisciplinary approach to decision making. Theories from psychoanalysis, neuro-psychoanalysis, neurology, and psychology are combined into a technical feasible system [6]-[8]. The main focus lies on theories from (neuro-)psychoanalysis. Thus, concepts like drives, emotions, desires, ego, id, and superego are used in this decision making module. Drives are a mapping of system (or bodily) needs [9]. For example, the need for energy could be modeled as a drive. The more energy is needed (and not already available) the stronger this particular drive is activated. If all drives are satisfied the system is in homeostasis. Emotions are general implicit situation assessments [10]. Desires are action plans to reach long term goals [11]. Ego, id, and superego are functional units with different tasks. The ego is responsible for basic and/or urgent system needs. It has to react on situations like very low energy or a dangerous situation like fire in the building. The superego is the center of social rules. It tells the system that for example, the room temperature has to be kept at about 21 degrees Celsius. The ego is responsible for planning and for conflict resolution. If the id wants to turn down heating due to lack of energy, but the superego tells the system to keep on heating, the ego has to decide whether heating should continue or not.

To facilitate the decision making, an episodic memory is introduced. This memory stores experienced situations. Based on the outcome of past situations, the decision making module is able to adapt its strategies. This is called learning by experience.

As test environments, the ARS-Project uses the SmartKitchen and the BubbleFamilyGame. The SmartKitchen is a kitchen equipped with many different sensors [12]. Currently, it is used to verify the implementation of the symbolization. The model developed by ARS-PA is inspired by the human brain. A test implementation for development purposes of this model into a building automation system is not possible. This is due to the fact that the theories from neuro-psychoanalysis, neurology, and psychology are aiming at a human being. Thus, an intermediate step - the BubbleFamilyGame-has been introduced. In this simulated environment autonomous agentsthe bubbles-roam around and try to find energy sources. Each agent is equipped with a neuro-psychoanalytically inspired decision making module. This intermediate step also makes it possible to implementation of this decision module into the autonomous robot Tinyphoon ${ }^{2}[13]$, [14] in future.

\section{EPISODIC MEMORY}

The episodic memory described in this article is based on the work of neuro-psychoanalysts [15] and psychologists [16] [19].

According to $[15$, p. 143], the human memory can be divided into a short term memory (alternatively: a working memory) and a long term memory. The short term memory stores information currently received or needed for further processing. Working memory is a module which temporarily stores and manipulates information. Thus, the working memory includes the short term memory. In contrast, the long term memory contains recent and old memories. The long term memory is divided into different units. They differ mainly by

\footnotetext{
${ }^{2}$ http://www.tinyphoon.com/
} 
the type of data they store and on their principles of operations $[17$, p. 13]. Typically, the human long term memory consists of three parts:

- Semantic Memory "Is a network of associations and concepts that underlies our basic knowledge of the worldword meanings, categories, facts, and propositions, and the like" [15, p. 150]. This memory stores world knowledge like that a table typically has four legs. It is accessed by a human if he can say "I know".

- Procedural Memory "Is a kind of bodily memory. It is memory for habitual motor skills, or, more generally, perceptuomotor or ideomotor skill" [15, p. 156]. Knowledge about periodically tasks like running or swimming is stored here.

- Episodic Memory "Involves the literal reexperiencing of past events-the bringing back to awareness of previous experiential episodes" [15, p. 160]. Here, the personal experiences like having been in school are stored. A human accesses this memory if he can say "I remember".

According to [15, pp. 140], the functions of the human episodic memory are (Figure 1 :

\begin{tabular}{|l|l|l|l|l|l|l|}
\hline Encoding & \\
\hline & Storage & Consolidation \\
\hline
\end{tabular}

Fig. 1. Functions of the Human Episodic Memory

- Encoding is the process of transforming a perceived event into a memory entry $[18$, p. 42]. According to [16, p. 37, p. 143], an event is something that occurs in a particular situation, is unique, and always has a beginning and an end. Not every event is transformed and stored. Only salient events are processed.

- Storage covers all processes responsible for maintenance of stored memory entries. Two important concepts are consolidation [18, p. 87] and forgetting [16, p. 203]. The first describes that memory entries which are related should be connected. Thus, important entries can be identified by their connectivity. The second defines the accessability and availability of memory entries. This is done by defining an activation level for each entry. The initial value depends on the salience of the stored entry. The less important an entry is and the longer it is not accessed, the lower this level gets by time.

- Retrieval is the process of fetching memory entries for further usage. This can happen either spontaneously or deliberately. Deliberate retrieval occurs when an explicit question is asked (e.g. "Where have I seen a red car the last time?"). During spontaneous retrieval, the currently processed event is used as query. The returned memory entries are used to complete the current perception with earlier made observations (e.g.: "I have seen a similar red car somewhere.").

The salience of an event is strongly influenced by the emotional state of a person [19, p. 276]. Thereafter, the decision of what to encode and store or the return results of the retrieval process depend on emotions. [15, p. 106] define an emotion as an internally directed sensory modality which is a state dependent function reflecting bodily changes in relation to the outer world. A detailed description of a technical model for emotions can be found at [10].

For a human, an episodic memory is essential. Without it, learning and reflecting on past experiences would not be possible for humans. But what benefits does an, in its functionality strictly limited, episodic memory bring to an embodied autonomous agent?

First of all, the episodic memory is the key to a huge variety of new functions that can be realized. In the following paragraphs, some essential functionalities will be described the agent can take advantage in its decision making process.

Since the episodic memory records events that have been experienced by the agent, it becomes also possible to reconstruct the way that brought the agent to the position in which it is located at the moment. This makes it also possible to walk the reverse path and go back to make the last actions undone, assuming the chain of events are reversible.

The content of the episodic memory makes the agent individual. Every agent has its own experiences in the past and therefore its own entries in the structure of the episodic memory. Being able to search for similar events or situations, the agent can compare the impact of different actions it tried in the past. The most pleasurable action-meaning that the internal values have been influenced most positively-can be the action that is selected as the next action. The episodic memory therefore can have an impact-positive or negativeto the stability of such agent internal values like the energy level, extending the lifespan of the agent.

Modern self localization algorithms as depicted in [20] make it possible to recognize landmarks and therefore determining the position within its environment by providing episodic memory. Typical, perceivable landmarks within the simulation environment described later are energy sources, obstacles, shapes of the local terrain, or other agents.

Acting within more complex and dynamic situations requiring knowledge of the context of past events or situations with social interaction between other agents makes episodic memory essential. It is also a first step towards a learning agent. This can be learning from past experiences, extending the world representation knowledge the agent is placed in, and generating semantic rules as a result of often observed circumstances.

As a last function that should be mentioned, experiences stored in the episodic memory can be shared between agents if they have the possibility to communicate with each others. In the next section, several related projects, which try to implement some of the mentioned features are introduced.

\section{StATe Of The ART}

There exists a lot of research on episodic memory (EM) in the scientific fields of neurology, psychology, and psychoanalysis. However, up to our knowledge, not many projects have focused on building a computational model of an EM which 
implements research findings of these sciences. This section presents an overview of previously done technical approaches to EM.

\section{A. Soar}

A computational episodic memory model is designed and integrated into the Soat ${ }^{3}$ cognitive architecture [21], [22]. The EM framework is based on the processing stages proposed by the psychologist Tulving [16]: encoding, storage, and retrieval. Episodes are stored as snapshots of working memory elements attached with an activation level [23]. For retrieval, the episode with the best match to a certain cue is selected. The EM model can be used for decision making. It is evaluated in a "Pacman"like game where autonomous agents navigate in a grid world. Although agents equipped with an EM outperform those which do not have an EM, the model has some limitations. Forgetting and spontaneous retrieval is not implemented. Among the stored episodes is no organization and the retrieval phase may be slow due to a linear search through all stored episodes. Furthermore, the influence of emotions on EM and interactions with semantic memory (SM) are not considered.

\section{B. Tecuci}

The work of Tecuci [24] addresses the organization of a generic EM model, the maintenance due to changes, and the retrieval of stored memories. New episodes are interpreted on the basis of expectations the system has of what will happen. SM is used for encoding of episodes and provides general knowledge about the application domain like performable actions or perceivable goals. Episodes are grouped by "memory organization packets" and are organized in a similarity network. Both deliberate and spontaneous retrieval is considered. A limitation of this model is that no forgetting mechanism is implemented and the tight coupling of memories with emotions is not considered.

\section{ISAC}

An EM system with emotions is addressed in [25] for a cognitive humanoid robot called ISAC4 Episodes are recorded in a time-indexed format and consist of various SM units. Emotions are used to give an experienced episode particular significance. The robot should remember emotional salient experiences very well. Furthermore, recently accessed episodes score higher and novel aspects are more important than common ones. These aspects are implemented by a history component, which is a time-dependent exponential decay function that depends on the emotional salience of an episode. Memories are removed if their history component gets below a certain cut-off value. This design of an EM is domain dependent.

\footnotetext{
${ }^{3}$ Soar-States, Operators, and Reasoning

${ }^{4}$ ISAC-Intelligent Soft Arm Control
}

\section{D. $\mathrm{Ho}$}

The goal of the work of Ho and Dautenhahn [26], [27] is to build a generic memory control architecture for autonomous agents to survive in a virtual environment. The agents have various physiological variables which have to be kept within a certain range. If one of the internal physiological variable gets out of its bound, an event reconstruction process is triggered. It is searched in the agents' autobiographical history for situations satisfying the agents' current internal needs. Events as series of situations can be reconstructed by reexecuting actions. This model of an EM is only applicable for navigation problems where agents have to find a "best path". No forgetting mechanism is implemented and emotions and semantic memories are not considered.

\section{ARChitecture}

The architecture for an episodic memory described in this section is based on the human episodic memory as described in Section III. Currently, this architecture is developed for simulated autonomous agents [28].

The sensor information is provided by the perceived images. They are a collection of already symbolized sensor data-the features. For further processing, a perceived image is compared to template images. Each of these templates describes a certain situation like on the left side not far away there is an energy source. A situation is defined as the composition of all information relevant to characterize the current state of an agent. The result of the matching is the probability that a template has been perceived. These values are used in further decision making processes.

The system is also able to detect scenarios, which are sequences of events. A scenario is defined by a state chart. The transition between two states is triggered by a template image match. There may be a large number of non fitting matches between two consecutive transitions. Once the final state of a scenario has been reached, the scenario is considered to be identified. The set of all events between the starting event and the final event is called episode. Figure 2 shows the correlation between a scenario and an episode. Scenario A consist of three states and two transitions triggered by template images (TI). Episode 1 starts with the perception of event 1. This event is the trigger for the scenario recognition to proceed to state 2 in scenario A. The next two perceived events $(2+3)$ are not important for this particular scenario. Finally, event 4 is perceived which triggers the second and final transition. Thus, the identified scenario $\mathrm{A}$ is the episode of four consecutive events.

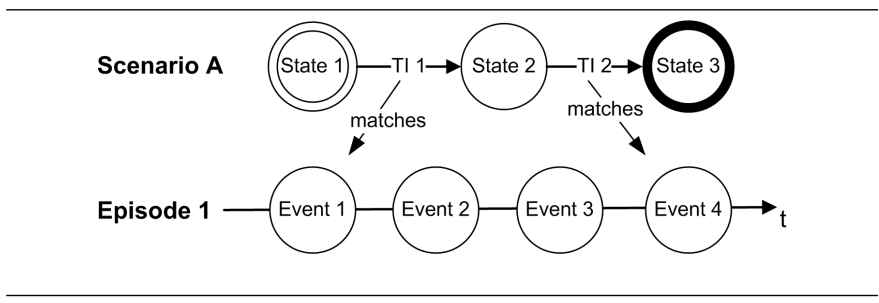

Fig. 2. Scenario and Episode 
As stated above, an event is only encoded and stored if its initial salience is above a threshold. Figure 3 shows the composition of an event and the corresponding salience. The elements of an event-TI-matches, emotional states, executed actions, etc.- - are grouped to features. Each feature selects the maximum salience of its elements. Together with a coefficient, they are summarized to the salience of this event. The salience for a feature element is determined for example by its unusualness, its novelty, its importance. For emotions, this would be a strong change of the value. The match probability is used for the TIs. The salience for actions is reciprocal to their endurance.

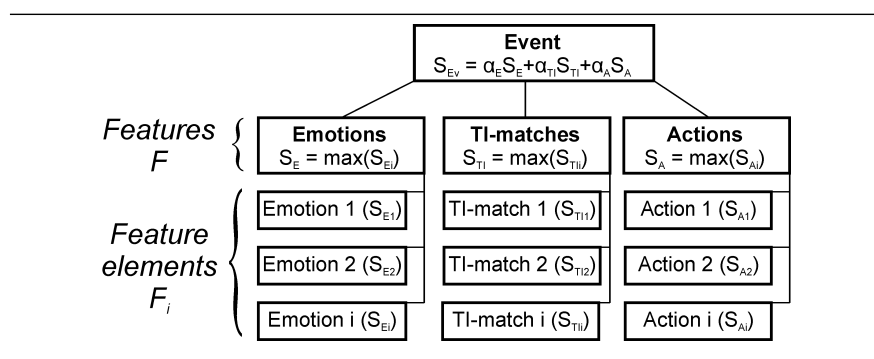

Fig. 3. Salience Structure

Each stored event may belong to none, one, or many episodes. Episodes may overlap or be nested.

Once an event is stored into the episodic memory, its salience declines with time. Hence the events are harder to recall-they are getting forgotten. According to [18, p. 73], the rate of forgetting slows down with time. [19, p. 220] states that the rate of correctly recalled events in relation to the time elapsed since they have been recorded decreases exponentially with time. Thus, in a first approach, we have modeled the forgetting rate as an exponential function. This curve is disturbed if the event is recalled (see Figure 4). The activation level or salience of the event starts with the value of 1 . Until the boost $A_{b 0}$ the level declines undisturbed. The better the event is consolidated and nested within episodes, the more often it is directly or indirectly recalled and thereafter boosted.

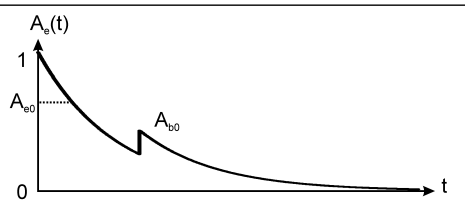

Fig. 4. Activation

To retrieve stored information from the episodic memory, the query has to be formulated as a cue. This is done by partially filling an event data structure. Each entry of the memory is compared with this cue. The match quality is determined by how many feature elements of cue are fitting to their counterpart elements of an entry. The matching events are ordered by their match quality together with the activation level. If the highest rated event is above a certain threshold, it is recalled. The retrieval cue can also be-next to an event-a scenario and previous or succeeding events.

As described above, retrieval of stored information can be done spontaneous or deliberative. While for deliberate retrieval the cue can be filled with all elements just described, for spontaneous retrieval only the currently perceived and encoded event is used as cue.

Only events can be recalled. If an event is part of an episode, previous and succeeding events of this episode can be fetched from memory (see Figure 5). This enables the anticipation of the outcome of the current situation. If a similar episode has been experienced, the outcome of it can be used for decision making.

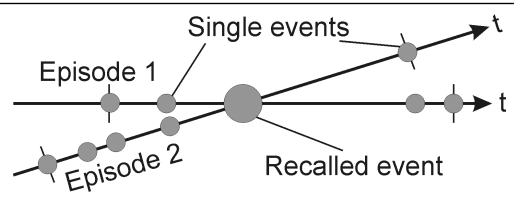

Fig. 5. Episode Reconstruction

The spontaneous retrieved results influence the emotional state of the agent. If a perceived event recalls an old event during which the agent was angry, the emotion anger will adopt towards this target value. This enables the agent to adapt its decision making to unclear situations.

\section{Evaluation}

To evaluate the performance of the implemented episodic memory model, a simulation environment for embodied, autonomous robots called bubble family game shown in Figure 6 was built. This test environment, inspired by the fungus eaters of [29], shall be described in detail in the following part.

The environment provides a playground limited in its dimension. It includes obstacles that can be shaped in triangles, rectangles, or circles. Different types of terrain are provided like grassland, steppe, desert, swamp, etc., influencing the behavior of the agent. Different types of energy sources (ES) can be created, satisfying different bodily needs.

Within this environment, different teams of agents (called bubbles), differing in their initial values and architecture like energy level, behavior rule set, knowledge base, and decision making unit are instantiated. The goal of each team as well as for the individual bubble is to survive as long as possible. This can be reached best by cooperation with team mate agents. Here, an episodic memory storing recently past episodes becomes an essential tool for survival.

To encourage cooperation between agents, several possibilities of teamwork is provided. One of the teamwork encouraging objects is an ES that can only be consumed by two agents cooperating like a huge elephant that can only be brought down by a group of hunters. Another circumstance is that agents can defend their location better from the enemy team when they stay within a group_like the safety within a flock. These possibilities are described in [30] in detail.

During simulation runs, it was shown that an episodic memory has positive effect to the time, the teams are surviving 
Bubble Family Game

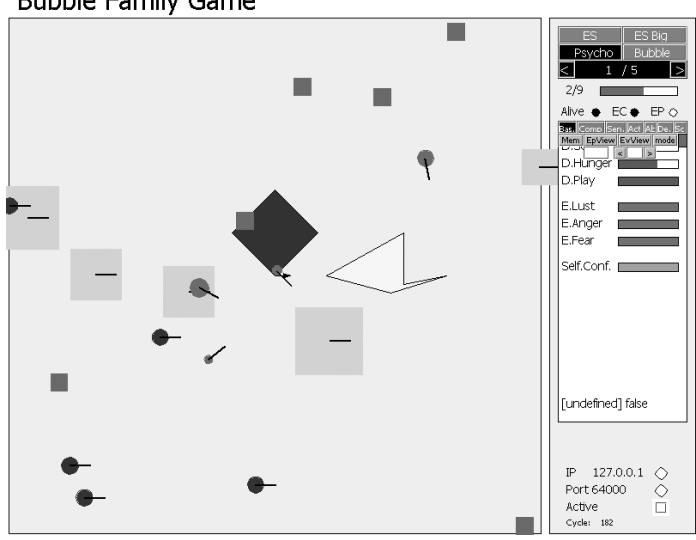

Fig. 6. Bubble Family Game

in their surrounding, using this sort of memory. Figure 6 shows the simulation environment with two different types of teams (circles), several obstacles (dark grey squares), five ES (light grey squares), and three different types of terrain. The right side of the picture contains the information panel, where the inner values and states of the selected agents can be viewed. In this panel, detailed analysis of the episodic memory module can be viewed.

Four scenarios were used for the evaluation:

1) Cooperation for Large ES-The agent is positioned at an ES that is only consumable with at least two agents. It calls another agent to make the ES consumable. Finally, the large ES is consumed.

2) Consume Small ES-An agent is above a consumable ES and consumes the energy.

3) Help to Crack Large ES-An agent receives a help call to open a large ES. It moves to the ES and helps the other agent.

4) Fight Against Enemy-An agent fights against an agent from another team.

During 20 spontaneous retrievals observed in various simulation runs, a retrieval accuracy of $80 \%$ has been measured. The retrieval accuracy is regarded as the effectiveness of the retrieval cue construction mechanism-whether the salient features of the currently encoded event serve as effective reminders for similar episodes.

In further 30 spontaneous retrievals observed in various simulation runs, the agents were able to anticipate $83 \%$ of the situations correctly. With this capability, the agents are able to foresee the impact of certain behaviors, which improves their decision making.

Agents with an episodic memory lived in average $20 \%$ longer than agents without. In more complex setups it is expected that this number will increase.

\section{CONCLUSIONS AND OUtLOOK}

We presented a new model and its implementation of an episodic memory for autonomous agents, inspired by neuropsychoanalytical and psychological concepts. After describing the underlying psychological model, the technical implementation was described. Evaluations within the described simulation software called the bubble family game showed that agents with episodic memory are positively effected in their variety of reactions and the time the agents are surviving by e.g. increased social activities including teamwork.

In future work, a semantic memory will be implemented that interacts with the episodic memory. As described in [16, p. 142], the episodic memory is tightly coupled with the semantic memory. Often experienced circumstances shall be abstracted to higher semantic rules and world representation knowledge.To cope with the huge amount of data that is stored in the episodic memory, different mechanisms of handling the increasing data shall be implemented and compared. A first approach is simply deleting data with a low activation level. As a second approach, memories can be condensed and optionally added to the world representation knowledge as described above. Outsourcing old data into other, possibly slower memory regions that are only accessed when no entry can be found in the normal memory region, can be a third possibility for data reduction.

\section{REFERENCES}

[1] G. Russ, "Situation-dependent behavior in building automation," Ph.D. dissertation, Vienna University of Technology, Institute of Computer Technology, 2003.

[2] G. Pratl and P. Palensky, "Project ARS - the next step towards an intelligent environment," Proceedings of the IEE International Workshop on Intelligent Environments, pp. 55-62, 2005.

[3] G. Pratl, D. Dietrich, G. P. Hancke, and W. T. Penzhorn, "A new model for autonomous, networked control systems," IEEE Transactions on Industrial Informatics, vol. 3, pp. 21-32, 2007.

[4] C. Tamarit, "Automation system perception - first step towards perceptive awareness," Ph.D. dissertation, Vienna University of Technology, Institute of Computer Technology, 2003.

[5] G. Pratl, "Processing and symbolization of ambient sensor data," Ph.D. dissertation, Vienna University of Technology, Institute of Computer Technology, 2006.

[6] E. Brainin, D. Dietrich, W. Kastner, P. Palensky, and C. Rösener, "Neurobionic architecture of automation systems : Obstacles and challenges," Proceedings of 2004 IEEE AFRICON, 7th Africon conference in Africa, Technology Innovation, vol. 2, pp. 1219-1222, 2004.

[7] T. Deutsch, R. Lang, G. Pratl, E. Brainin, and S. Teicher, "Applying psychoanalytic and neuro-scientific models to automation," The 2nd IET International Conference on Intelligent Environments, pp. 111-118, 2006.

[8] C. Rösener, B. Lorenz, G. Fodor, and K. Vock, "Emotional behavior arbitration for automation and robotic systems," Proceedings of 2006 IEEE International Conference of Industrial Informatics, pp. 423-428, 2006.

[9] A. Damasio, Looking for Spinoza: Joy, Sorrow, and the Feeling Brain. Harvest Books, 2003, iSBN 0-15-100557-5.

[10] W. Burgstaller, R. Lang, P. Pörscht, and R. Velik, "Technical model for basic and complex emotions," Proceedings of 2007 IEEE International Conference of Industrial Informatics, 2007.

[11] C. Rösener, R. Lang, T. Deutsch, and A. Gruber, "Action planning model fpr autonomous mobile robots," Proceedings of 2007 IEEE International Conference of Industrial Informatics, 2007.

[12] S. Goetzinger, "Scenario recognition," Master's thesis, Technische Universität Wien, Institut für Computertechnik, 2006.

[13] G. Novak, "Multi agent systems robot soccer," Ph.D. dissertation, Technische Universität Wien, Institut für Handhabungsgeräte und Robotertechnik, 2002.

[14] G. Novak, C. Roesener, M. Bader, T. Deutsch, S. Jakubek, S. Krywult, and M. Seyr, "The tinyphoons control concept," Proceedings of ICM 2006, IEEE 3rd International Conference on Mechatronics, 3.-5. Juli 2006, Budapest, Ungarn, 2006., pp. 625-630, 2006. 
[15] M. Solms and O. Turnbull, The Brain and the Inner World. Karnac/Other Press, Cathy Miller Foreign Rights Agency, London, England, 2002, iSBN 1-59051-017-8.

[16] E. Tulving, Elements of Episodic Memory. Oxford: Clarendon Press, 1983, iSBN 0198521022.

[17] D. L. Schacter and E. Tulving, Memory Systems. The MIT Press, 1994, iSBN 0262193507.

[18] D. L. Schacter, Searching for Memory: The Brain, the Mind, and the Past. Basic Books, 1996, iSBN 0-465-02502-1.

[19] A. Baddeley, Human Memory: Theory and Practice. Psychology Press, 1997, iSBN 0-86377-431-8.

[20] D. Fox and S. Thrun, Probabilistic Robotics. MIT Press, 2006, iSBN 0-262-20162-3

[21] A. Nuxoll and J. E. Laird, "Enhancing intelligent agents with episodic memory," University of Michigan, Artificial Intelligence Laboratory, Tech. Rep., 2002.

[22] _ "A cognitive model of episodic memory integrated with a genera cognitive architecture," International Conference on Cognitive Modeling, pp. 220-225, 2004.

[23] A. Nuxoll, J. E. Laird, and M. R. James, "Comprehensive working memory activation in soar," Proceedings of the 6th International Conference on Cognitive Modeling, pp. 226-230, 2004.

[24] D. Tecuci, "A generic episodic memory module," University of Texas at Austin, Tech. Rep., 2005.

[25] W. Dodd and R. Gutierrez, "The role of episodic memory and emotion in a cognitive robot," IEEE International Workshop on Robots and Human Interactive Communication, pp. 692-697, 2005.

[26] W. C. Ho, K. Dautenhahn, C. L. Nehaniv, and R. T. Boekhorst, "Sharing memories: An experimental investigation with multiple autonomous autobiographic agents," IAS-8, 8th Conference on Intelligent Autonomous Systems, pp. 361-370, 2004.

[27] W. C. Ho, K. Dautenhahn, and C. L. Nehaniv, "Autobiographic agents in dynamic virtual environments - performance comparison for different memory control architectures," IEEE Congress on Evolutionary Computation, pp. 573-580, 2005

[28] A. Gruber, "Neuro-psychoanalytically inspired episodic memory for autonomous agents," Master's thesis, Technische Universität Wien, Institu für Computertechnik, 2007.

[29] M. Toda, Man, Robot and Society. Martinus Nijhoff Publishing, 1982 iSBN 089838060X.

[30] T. Deutsch, H. Zeilinger, and R. Lang, "Simulation results for the ars-pa model," Proceedings of 2007 IEEE International Conference of Industrial Informatics, 2007.

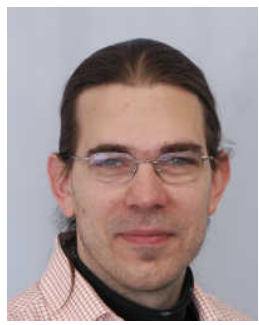

Section Austria.
Tobias Deutsch (M'06) was born 1975 in Vienna/Austria. After graduating at the HTL Ungargasse he started studying Computer Science at the TU Vienna in 1996 and completed his degree in March 2007. Since October 2005 he is staff member of the ICT. His current research focuses on cognitive intelligence, building automation and autonomous agents on the Vienna University of Technology at the Institute of Computer Technology. He is IEEE Member, Organizing Committee member of the IN DIN2007 and Treasurer of the IEEE Student Branch,

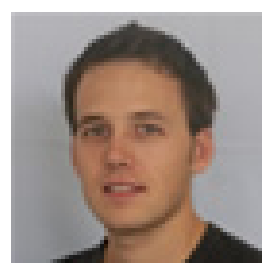

Andreas Gruber Andreas Gruber was born 1980 in Kirchdorf/Austria. After graduating at the HTL Steyr he started studying electronic engineering and business economics at the TU Vienna in 2000 and completed his degrees in June 2007. Since October 2007 he is staff member of TTTech Computertechnik AG.

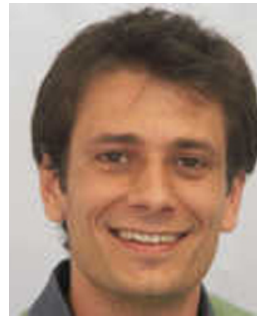

Roland Lang (M'06) was born 1978 in Vienna/Austria. He finished his studies of electronics, especially control engineering and telecommunications in June 2001 at the University of Applied Sciences Technikum Wien (Fachhochschule). He is currently employed at the Institute of Computer Technology and has gained experience on cognitive automation (within the project ARS - Artificial Recognition System). He is IEEE Member, Organizing Committee member of the INDIN2007 and Secretary of the IEEE Student Branch, Section

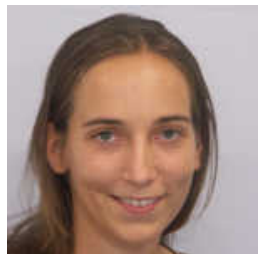

Rosemarie Velik (M'06) Rosemarie Velik was born in Sept. 1981 in Klagenfurt. After graduating at the HTL for Computer and Internet Engineering in Klagenfurt she began studying Electrical and Automation Engineering at the TU Vienna in Oct. 2001 and completed her degree in April 2006. Since July 2006 she is a member of the department staff and works in the Project ARS (Artificial Recognition System). Her research interests are devoted to the topic of building automation, bionics, neural networks, cognitive intelligence, and artificial percep- 